\title{
Right lung ischemia induces contralateral pulmonary vasculopathy in an animal model
}

Edouard Sage, MD, Olaf Mercier, MD, Philippe Herve, MD, Ly Tu, PhD, Philippe Dartevelle, MD, Saadia Eddahibi, $\mathrm{PhD}$, and Elie Fadel, MD

Objective: The study objective was to determine whether the vasculopathy seen in nonobstructed lung regions in chronic thromboembolic pulmonary hypertension is induced by the local blood flow increase or by factors released by the ischemic lung.

Methods: Three groups of 10 piglets were studied 5 weeks after right pulmonary artery ligation, right pneumonectomy, or right pulmonary artery dissection (sham). Pulmonary vascular resistance, pulmonary arterial vasoreactivity, and morphometry were measured, and gene expressions of factors involved in vascular smooth muscle cell proliferation were quantified.

Results: Left lung blood flow was similarly increased after right pneumonectomy and right pulmonary artery ligation. Compared with right pneumonectomy, right pulmonary artery ligation resulted in left lung vasculopathy with increased pulmonary vascular resistance $(P=.0009)$, medial hypertrophy of the distal pulmonary artery $(P<.0001)$, and decreases in maximal relaxation to acetylcholine $(P=.013)$ and endothelial nitric oxide synthase gene expression $(P=.041)$. These values were similar after sham and right pneumonectomy. In the left lung, right pulmonary artery ligation increased the gene expressions for insulin-like growth factor $(P=.034)$, platelet-derived growth factor $(P=.0006)$, and vascular endothelial growth factor $(P=.0105)$ compared with right pneumonectomy and sham. Whereas endothelin-1 gene expression was not affected, expressions of endothelin-1 receptors A and B were downregulated after right pneumonectomy $(P=.048$ and $P=.039$, respectively) and right pulmonary artery ligation $(P=.033$ and $P=.028$, respectively).

Conclusions: Pulmonary vasculopathy was absent in the remaining lung 5 weeks after right pneumonectomy but developed in the nonobstructed lung regions 5 weeks after right pulmonary artery ligation, suggesting that factors released by the ischemic lung induced vascular remodeling in the contralateral lung. This endocrine process may involve the release of factors involved in vascular smooth muscle cell proliferation. (J Thorac Cardiovasc Surg 2012;143:967-73)

Chronic thromboembolic pulmonary hypertension (CTEPH) is a rare and life-threatening disease in which organized thromboemboli obstruct the pulmonary arteries (PAs) and pulmonary vascular remodeling develops in the nonobstructed lung regions. These abnormalities increase the pulmonary vascular resistance, causing pulmonary hypertension $(\mathrm{PH})$ and progressive right-sided heart failure. The mechanisms underlying the vascular remodeling in the nonobstructed PAs are unknown.

After acute pulmonary embolism, the obstructed vascular territories are characterized by chronic ischemia and the

From the Laboratoire de Recherche Chirurgicale and INSERM U999, Hôpital Marie Lannelongue, Université Paris Sud, Le Plessis Robinson, France.

Disclosures: Authors have nothing to disclose with regard to commercial support.

Read at the 37th Annual Meeting of the Western Thoracic Surgical Association, Colorado Springs, Colorado, June 22-25, 2011.

Received for publication June 25, 2011; revisions received Dec 2, 2011; accepted for publication Dec 28, 2011; available ahead of print Jan 30, 2012.

Address for reprints: Elie Fadel, MD, Hôpital Marie-Lannelongue (Paris-Sud University), 133 Avenue de la Resistance, 92350 Le Plessis Robinson, France (E-mail: fadel@ccml.com).

$0022-5223 / \$ 36.00$

Copyright (c) 2012 by The American Association for Thoracic Surgery

doi:10.1016/j.jtcvs.2011.12.052 nonobstructed vascular territories are exposed to high shear stress resulting from redistribution of the pulmonary blood flow. ${ }^{1}$ Whether the pulmonary vascular remodeling is related to increased pulmonary blood flow has been investigated in various animal models. Consistent with data reported by several authors, ${ }^{2-4}$ our previous study in a pig model demonstrated that increased pulmonary blood flow for 5 weeks due to an aortopulmonary shunt induced pulmonary vasculopathy with medial hypertrophy of the distal PAs caused by smooth muscle cell (SMC) proliferation, impaired endothelium-dependent vasorelaxation, and increased expression of endothelin (ET)-1, ET receptor A (ETA), angiopoietin-1, angiopoietin-2, and Tie-2 in the lung parenchyma. ${ }^{1}$ However, these animal models do not accurately replicate human CTEPH because they do not induce chronic lung ischemia, which may be involved in the development of vasculopathy in the nonobstructed lung territories.

We hypothesized that endocrine factors released by the ischemic lung might account for the development of pulmonary vasculopathy in nonobstructed regions. To test this hypothesis, we studied the effects of chronic high blood flow due to cardiac output redistribution to the left lung after 


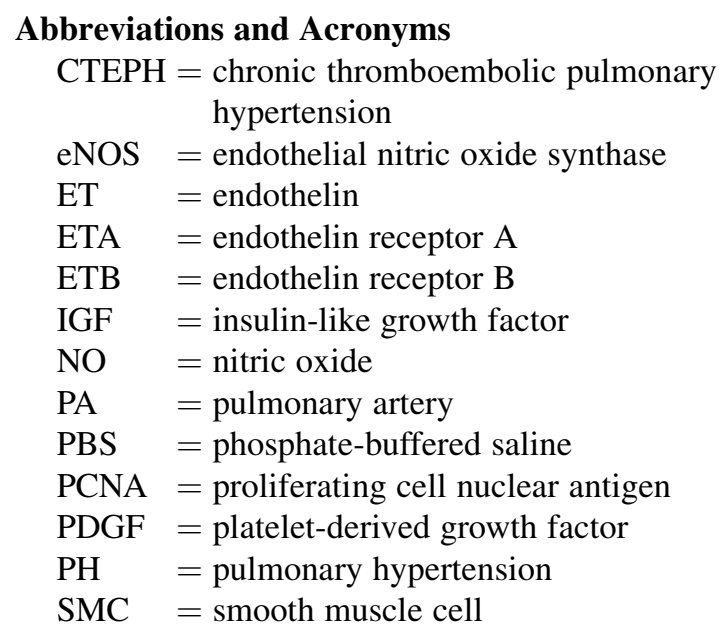

right pneumonectomy or right PA ligation in pigs to model blood flow redistribution combined with contralateral ischemia. Sham-operated animals served as controls. We investigated the angiogenic, ET, and nitric oxide (NO) pathways to look for underlying pathophysiologic mechanisms.

\section{MATERIALS AND METHODS}

Thirty Large White piglets weighing $22 \pm 4 \mathrm{~kg}$ were used. The study complied with the Principles of Laboratory Animal Care developed by the National Society for Medical Research, and the Guide for the Care and Use of Laboratory Animals written by the Institute of Laboratory Animal Resources and published by the National Institutes of Health (publication no. 86-23, revised in 1985).

\section{Study Groups}

The piglets were randomly allocated to 3 groups of 10 animals each. The studies were done 5 weeks after right PA ligation (ligated group), right pneumonectomy (pneumonectomy group), or a sham operation consisting only in dissection of the right PA (sham group).

\section{Surgical Procedure}

Anesthesia was induced with intramuscular ketamine $(100 \mathrm{mg} / \mathrm{kg})$ and maintained with intravenous pentobarbital $(10 \mathrm{mg} / \mathrm{kg}$ bolus, followed by a continuous infusion of $0.1 \mathrm{mg} / \mathrm{kg} / \mathrm{min}$ ). The animals were paralyzed with pancuronium $(0.3 \mathrm{mg} / \mathrm{kg})$. After endotracheal intubation, intermittent positive-pressure ventilation was provided (MMS RET 107 ventilator; MMS, Pau, France) at a tidal volume of $15 \mathrm{~mL} / \mathrm{kg}$, with a respiratory rate of 18 cycles/min and a fraction of inspired oxygen of 0.5 . Body temperature was maintained at $37^{\circ} \mathrm{C}$.

With the animal in the supine position, a midline sternotomy was performed under sterile conditions, and the pericardium was opened. For right PA ligation, the right PA was approached after retracting the superior vena cava and mobilizing the ascending aorta and was ligated using a nonabsorbable loop. For right pneumonectomy, the same approach was used and the right PA was ligated and divided. The right pleura was then opened to allow division of the upper and lower pulmonary veins and right bronchus. The right lung was removed without chest drainage. In the sham group, the approach was the same but the right PA was merely dissected. After the procedure, the chest was closed and the animal was allowed to recover.

\section{Tissue Preparation}

Five weeks later, the pigs were anesthetized as described above. A midline sternotomy was performed. After heparin administration, the animal was killed by exsanguination and the left lung was rapidly removed from the chest. The left PA was then flushed with $500 \mathrm{~mL}$ of $0.9 \%$ normal saline solution. Biopsy specimens weighing 300 to $500 \mathrm{mg}$ were taken from the left upper lobe, snap-frozen in liquid nitrogen, and stored at $-70^{\circ} \mathrm{C}$ or fixed in $4 \%$ paraformaldehyde solution.

\section{Hemodynamic Measurements}

Pulmonary and systemic hemodynamic variables were measured before the animals were killed. Aortic and pulmonary blood flow were measured using a flow probe (Transonic Systems, Inc, Ithaca, NY) placed on the origins of the aorta and pulmonary trunk. Pressures and blood gases were measured by direct puncture at the origins of the aorta and left PA. Total pulmonary resistance was calculated as mean PA pressure divided by flow (Q).

\section{Light Microscopy and Morphometry}

Fixed lung sections were processed using standard histologic techniques and embedded in paraffin. We sought to identify 30 to 40 arteries less than $200 \mu \mathrm{m}$ in diameter in each piglet. Medial thickness (MT) was calculated as follows:

$$
\% \mathrm{MT}=(\mathrm{ED}-\mathrm{ID}) \times 100 / \mathrm{ED}
$$

where ED is the external diameter and ID the internal diameter.

\section{Evaluation of In Situ Pulmonary Artery Small Muscle Cell Proliferation}

To assess the PA-SMC proliferation, the proliferating cell nuclear antigen (PCNA) was evaluated. Tissue sections were deparaffinized in xylene, treated with a graded series of alcohol washes, rehydrated in phosphatebuffered saline (PBS) ( $\mathrm{pH} 7.5$ ), and incubated with target retrieval solution (Dako, Trappes, France) into a water bath at $90^{\circ} \mathrm{C}$ for 20 minutes. Endogenous peroxidase activity was blocked using hydrogen peroxide in PBS $(3 \% \mathrm{vol} / \mathrm{vol})$ for 5 minutes. Slides were then washed in PBS and incubated for 30 minutes in a protein-blocking solution consisting of PBS supplemented with $3 \%$ bovine serum albumin. The slides were subsequently incubated for 30 minutes with anti-PCNA mouse monoclonal antibody (PC-10, 1:200; Dako, France). Antibodies were washed off and the slides were processed using the alkaline phosphatase LSAB+System-HRP Detection Kit (DAKO, Carpinteria, Calif). Brown color was generated by using a diaminobenzidine substrate, and nuclei were counterstained with hematoxylin.

\section{Pulmonary Artery Reactivity}

At the end of the study, intrapulmonary arterial segments were dissected out and endothelial relaxation was investigated as described previously. ${ }^{5}$ Acetylcholine hydrochloride, calcium ionophore A23187, and sodium nitroprusside were used for relaxation after phenylephrine precontraction. The drugs used to assess PA reactivity were purchased from Sigma Chemical Company (St Louis, Mo).

\section{Real-Time Quantification by Polymerase Chain Reaction Assay}

Real-time polymerase chain reaction was conducted as previously described. ${ }^{6}$ Except for platelet-derived growth factor (PDGF) and insulin-like growth factor (IGF), we used specific porcine primers previously described by Rondelet and associates ${ }^{6}$ (vascular endothelial growth factor [VEGF], ET-1, ET-1 receptors ETA and ETB, and endothelial nitric oxide synthase [eNOS]). The primers for IGF-1 and PDGF had been used in 
our laboratory for human experimentation and were designed on Primer Express software (Applied Biosystems, Foster City, Calif).

To avoid inappropriate amplification of residual genomic DNA, intronspanning primers were selected and internal control 18S rRNA (r18S) primers provided. For each sample, the amplification reaction was performed in duplicate using SYBR Green mix (Applied Biosystems) and specific primers. Signal detection and result analysis were achieved using ABI-Prism 7000 sequence detection software (Applied Biosystems). Expression of the gene of interest was computed relative to expression of the internal standard mRNA, r18S, using the following formula: relative $\mathrm{mRNA}=1 / 2^{\text {(Ctgene of interest-Ctr18S) }}$.

\section{Statistical Analysis}

Data are described as means \pm standard error of the mean. Analysis of variance followed by the Fisher test for multiple comparisons was performed using the StatView software package version 5 (Abacus Concept, Berkeley, Calif).

\section{RESULTS}

\section{Hemodynamic Variables}

None of the piglets died prematurely. Heart rate and mean arterial pressure were not significantly different across the groups. Mean PA pressure was significantly elevated in the ligated group $(16.2 \pm 1.3 \mathrm{~mm} \mathrm{Hg})$ compared with the pneumonectomy $(11.6 \pm 0.7 \mathrm{~mm} \mathrm{Hg} ; P=.0069)$ and sham $(13.0 \pm 0.7 \mathrm{~mm} \mathrm{Hg} ; P=.0447)$ groups; this variable was not significantly different between the sham and pneumonectomy groups.

Mean absolute cardiac output was significantly decreased in the ligated group $(1.53 \pm 0.06 \mathrm{~L} / \mathrm{min})$ compared with the sham group $(2.04 \pm 0.13 \mathrm{~L} / \mathrm{min} ; P=.0265)$ but was not different between the ligated and pneumonectomy groups (1.8 $\pm 0.23 \mathrm{~L} / \mathrm{min})$.

Total pulmonary resistance was significantly higher in the ligated group $(10.05 \pm 0.69$ Wood units) than in the pneumonectomy $(6.83 \pm 0.51$ Wood units; $P=.0009)$ and sham $6.48 \pm 0.59$ Wood units; $P=.0004)$ groups. There was no significant difference between the sham and pneumonectomy groups.

\section{Pulmonary Vascular Remodeling in the Left Lung}

In the sham group, medial wall thickness of PAs less than $200 \mu \mathrm{m}$ in diameter was $22.9 \% \pm 0.7 \%$. Five weeks after right PA ligation, medial wall thickness was significantly increased compared with the pneumonectomy $(42.3 \% \pm$ $1.1 \%$ vs $21.8 \% \pm 0.7 \% ; P<.0001)$ and sham $(42.3 \% \pm$ $1.1 \%$ vs $22.9 \% \pm 0.7 \% ; P<.0001)$ values. Medial wall thickness was not significantly changed after pneumonectomy compared with the sham procedure $(21.8 \% \pm 0.7 \%$ vs $22.9 \% \pm 0.7 \% ; P=.29$ ). Plexiform lesions were not observed in any group (Figures $1, A$ and $B$, and 2).

PCNA labeling demonstrated a significant proliferation of SMCs in the distal PA wall in ligated animals. Five weeks after right PA ligation, the number of PCNA positive cells increased markedly in the left lung when compared with sham values $(32.4 \% \pm 11 \%$ vs $8 \% \pm 9 \%, P<.05)$.
Conversely, 5 weeks after right pneumonectomy, the number of PCNA positive cells in the remaining lung $(13 \% \pm$ $11 \%, P=$ not significant) was similar to sham values (Figure 2).

\section{Pulmonary Vascular Reactivity and Endothelial Function of the Left Lung}

Contraction of PA rings in response to phenylephrine was similar in all 3 groups. The relaxation response to sodium nitroprusside was not affected by right pneumonectomy or right PA ligation. Maximal relaxation in response to acetylcholine was significantly decreased in the ligated group compared with the pneumonectomy $(60.3 \% \pm 4.6 \%$ vs $83.5 \% \pm 3.5 \% ; P=.0157)$ and sham $(60.3 \% \pm 4.6 \%$ vs $91.3 \% \pm 5.9 \% ; P=.0139)$ groups. Pneumonectomy did not significantly modify maximal relaxation in response to acetylcholine $(83.5 \% \pm 3.5 \%$ vs $91.3 \% \pm 5.9 \%$; $P=.31$ ) (Figure 3).

Expression of Genes Involved in the Endothelin-1, Nitric Oxide Synthase, and Angiogenesis Pathways in the Left Lung

Compared with the sham group, the ligated group exhibited significant downregulation of eNOS gene expression $(P=.041)$. A trend toward decreased eNOS mRNA levels was found in the pneumonectomy group. There was no significant difference between the pneumonectomy and ligated groups (Figure 4).

Whereas ET-1 gene expression was not affected by the experimental procedures, ETA and ETB expressions were similarly downregulated in the pneumonectomy group ( $P=.048$ and $P=.039$, respectively) and the ligated group $(P=.033$ and $P=.028$, respectively) compared with the sham group (Figure 5).

As shown in Figure 6, left lung PDGF and VEGF gene expressions were increased in the pneumonectomy group compared with the sham group. The expression of these proangiogenic factors was significantly greater in the ligated group than in the pneumonectomy and sham groups. Left lung IGF gene expression was increased in the ligated group compared with both the sham and the pneumonectomy groups.

\section{DISCUSSION}

We found that right PA ligation for 5 weeks induced vascular remodeling in the left lung with increases in pulmonary vascular resistance and medial thickness of distal PAs, impaired endothelium-dependent vasorelaxation, and decreased eNOS gene expression. By contrast, 5 weeks after right pneumonectomy, no evidence of pulmonary vasculopathy was found in the left lung, where the hemodynamics, morphology, and NO pathway were similar to those in the sham animals. Of note, ligation was followed by upregulation of the expressions of the proangiogenic 

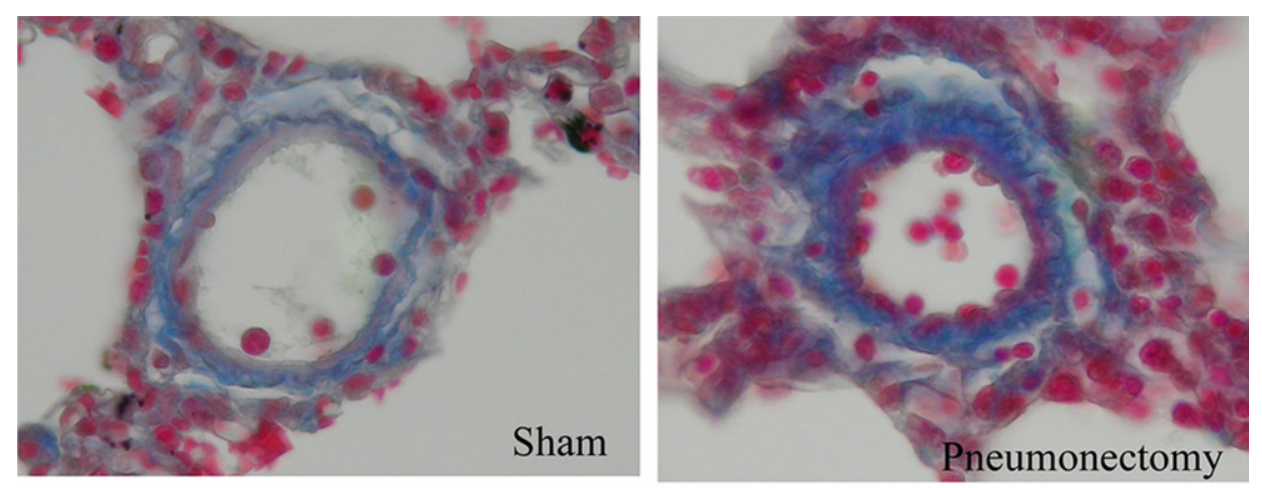

A
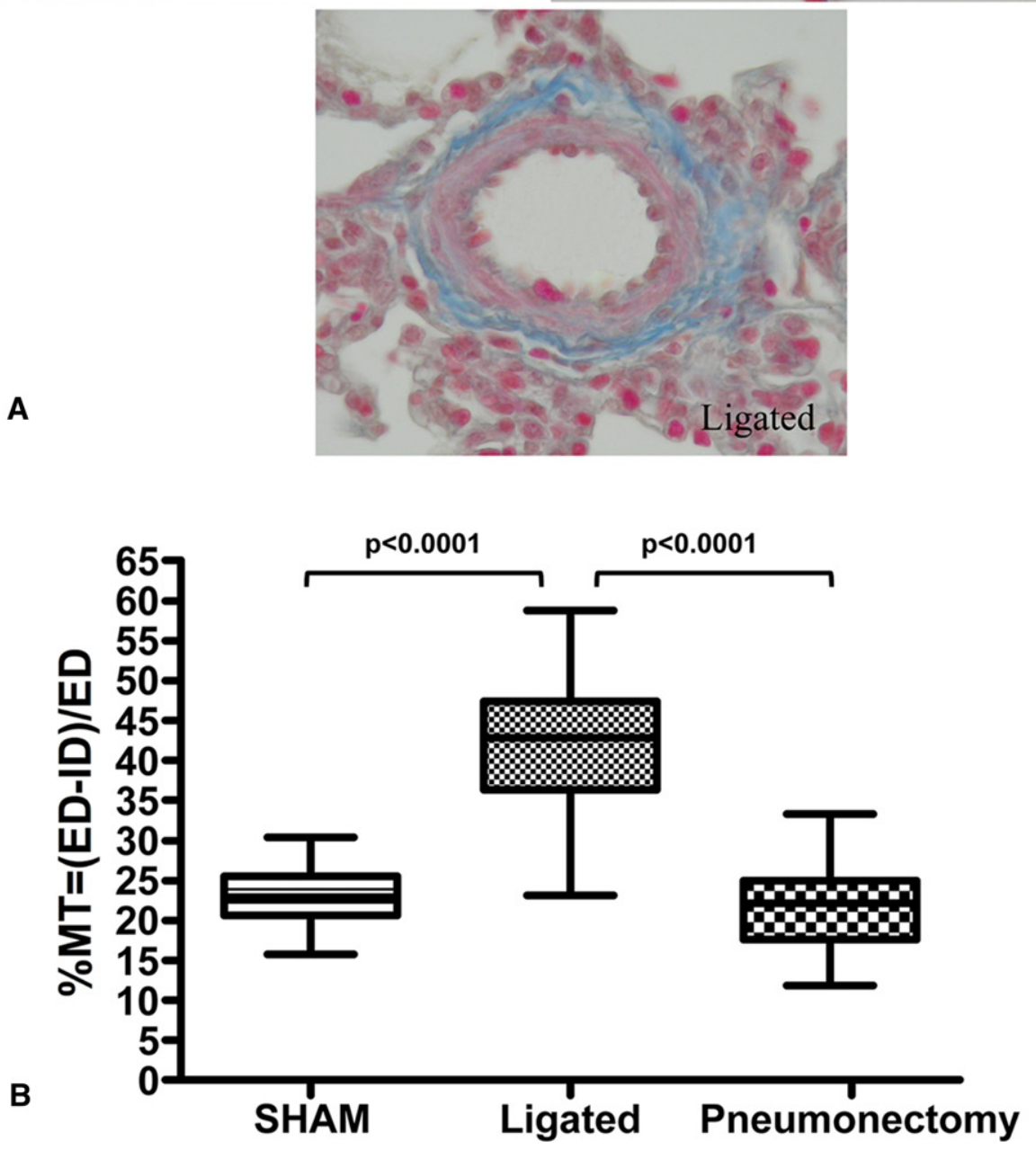

FIGURE 1. A, Morphometry $(\times 400)$ of PAs less than $200 \mu \mathrm{m}$ diameter in the sham, pneumonectomy group, and ligated groups. The percentage of media thickness was highest in the ligated group $(P<.05)$. B, Box plot of the percentage of media thickness of small PAs $(<150 \mu \mathrm{m}) 5$ weeks after a sham procedure, right PA ligation, or right pneumonectomy. $M T$, Medial thickness; $E T$, external diameter; $I D$, internal diameter.

factors IGF, PDGF, and VEGF. Because elevated shear stress affected the left lung similarly after right PA ligation and right pneumonectomy, this result suggests that the ischemic lung released factors that induced vascular remodeling in the nonobstructed PA tree, thereby contributing to increase the pulmonary vascular resistance.

CTEPH typically develops after 1 or more episodes of pulmonary embolism due to deep vein thrombosis. ${ }^{7}$ Failure of the emboli to resolve leads to segmental obstruction of the corresponding vascular areas with blood redistribution to nonobstructed areas and the progressive development of PH. The structural changes that are thought to underlie the increased pulmonary vascular resistance in CTEPH can be broadly classified as related to 2 different processes: (1) a reduction in the total number of blood vessels in the lung and (2) remodeling of the nonobstructed pulmonary 


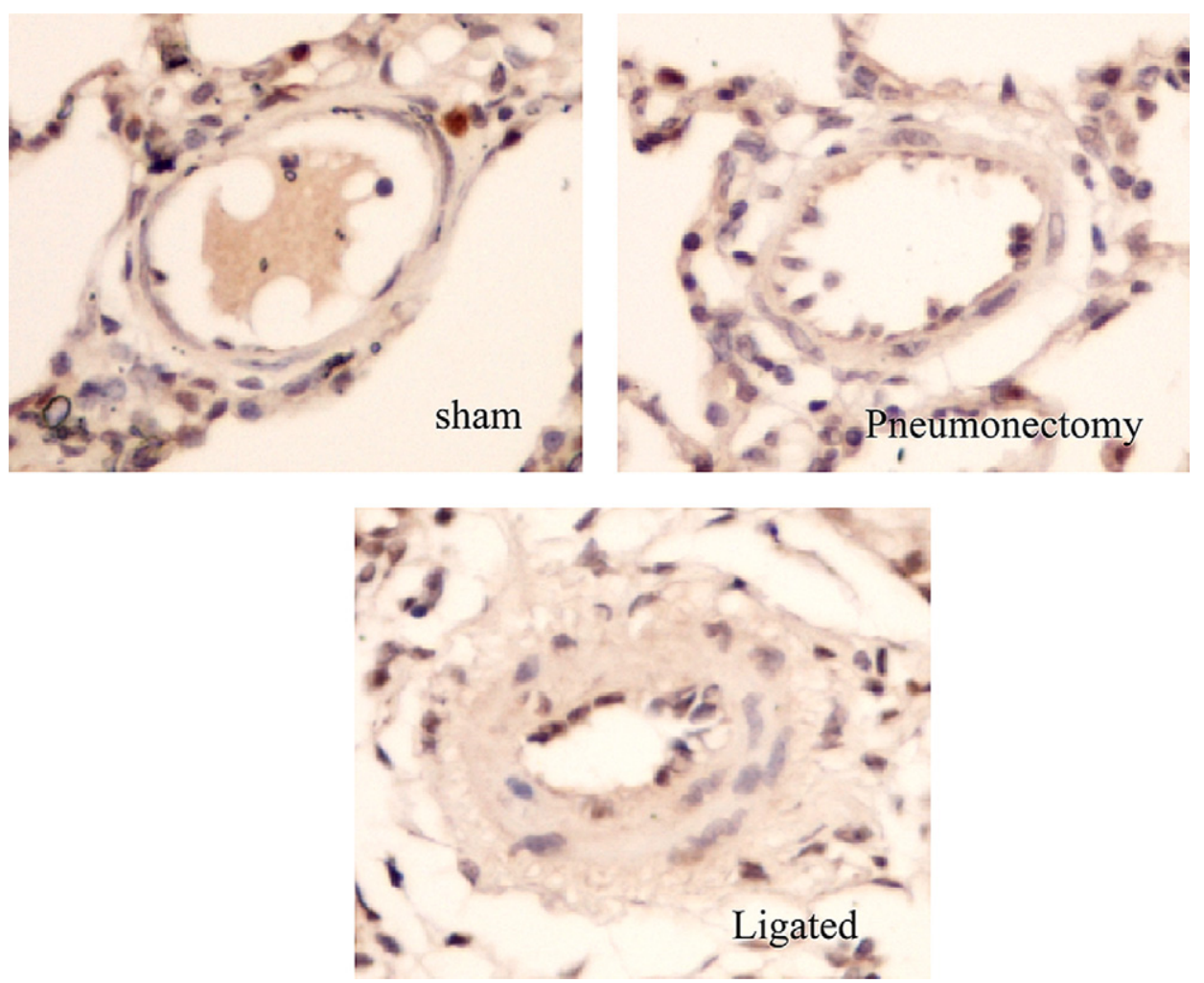

FIGURE 2. Evaluation of in situ PA-SMC proliferation as assessed by PCNA coloration in the left lung of sham, ligated, and pneumonectomy groups. PA wall thickness in the left lung 5 weeks after right PA ligation is related to the proliferation of SMCs.

resistance vessels. ${ }^{1,7-10}$ Two mechanisms have been suggested to explain the development of distal vasculopathy in nonobstructed regions, namely, prolonged excessive shear stress due to blood flow redistribution and chronic vascular release of proinflammatory and angiogenic mediators by ischemic lung tissue. To

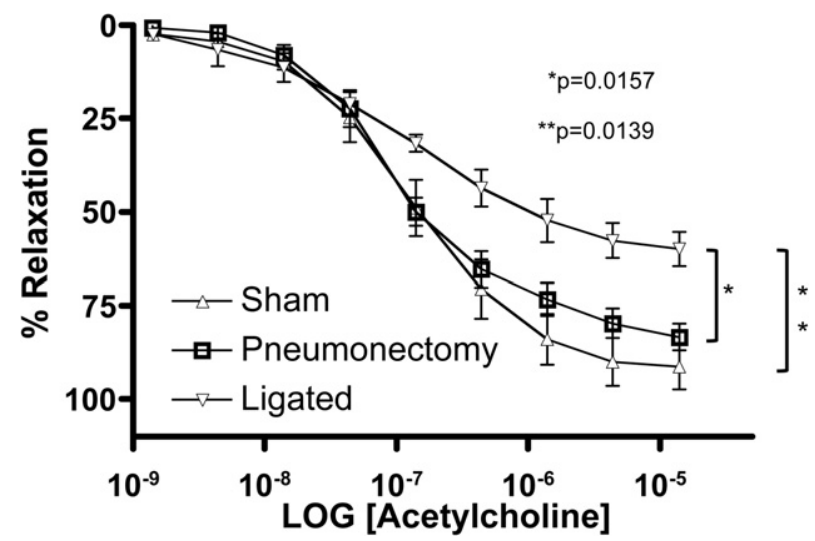

FIGURE 3. Vasoconstriction to ET-1 and relaxation to acetylcholine of PA rings from the sham, ligated, and pneumonectomy groups. Ligation of the right PA for 5 weeks induced a decrease in maximal relaxation to acetylcholine in the contralateral lung. In contrast, 5 weeks after right pneumonectomy left lung maximal relaxation was similar to that in sham-operated animals. investigate the respective roles for these 2 mechanisms, we compared 2 animal models characterized by chronic shear stress elevation without (right pneumonectomy) or with (right PA ligation) lung ischemia. Consistent with previous animal studies and clinical experience, $\mathrm{PH}$ and pulmonary vascular remodeling did not occur after right pneumonectomy in our study. ${ }^{11-13}$ Indeed, pneumonectomy directs the entire cardiac output to the

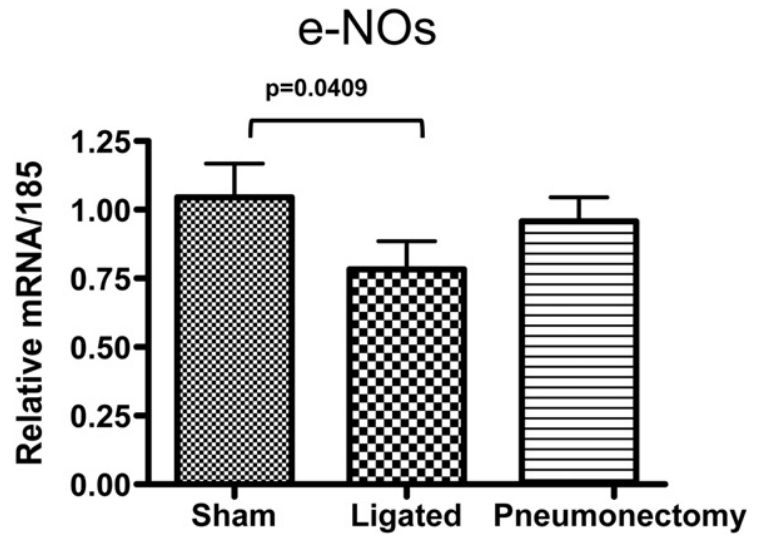

FIGURE 4. eNOS mRNA quantification in the left lung parenchyma by real-time polymerase chain reaction. Expression of eNOS was decreased 5 weeks after right $P A$ ligation but remained unchanged 5 weeks after right pneumonectomy. eNOS, Endothelial nitric oxide synthase. 


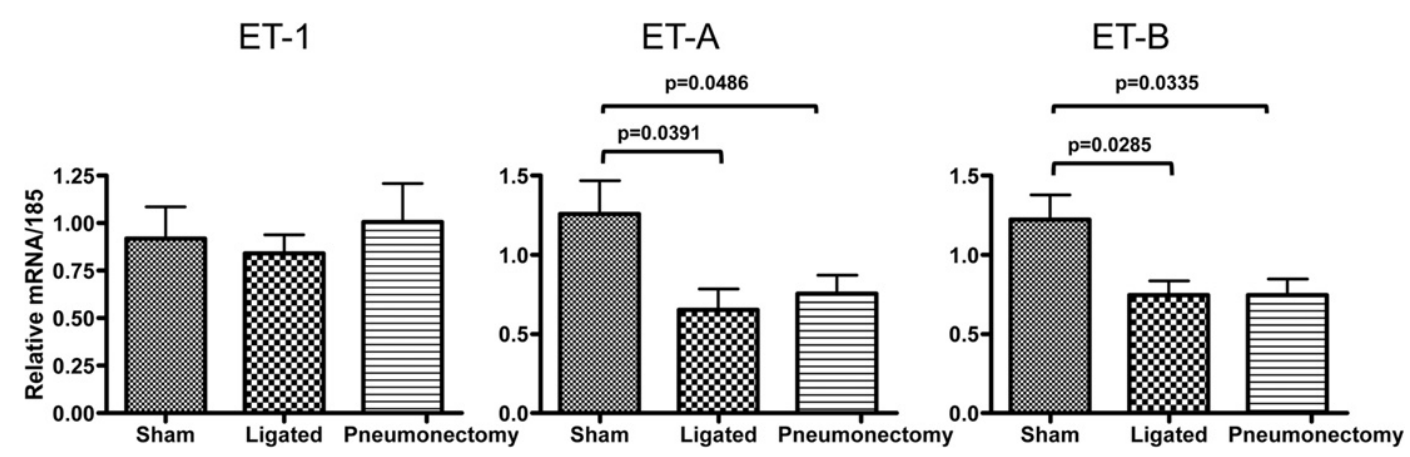

FIGURE 5. Quantification of mRNAs for ET-1 and its receptors (ETA and ETB) in left lung parenchyma by real-time polymerase chain reaction. Whereas ET-1 expression remained unchanged, ETA and ETB mRNA levels were decreased in the contralateral lung 5 weeks after right PA ligation or right pneumonectomy. ET-1, Endothelin-1; ETA, endothelin receptor A; ETB, endothelin receptor B.

remaining lung resulting in transient increase of PA pressure, and in under normal circumstances, this value returns to baseline level despite amputation of half of the PA bed. ${ }^{11}$ Several adaptive mechanisms protect the pulmonary vascular bed from the consequences of increased shear stress under these conditions, including vascular dilatation and distention involving upregulation of the NO pathway in the first 2 postoperative weeks. ${ }^{11}$ Our finding of normal eNOS expression 5 weeks after pneumonectomy confirms the transient role for $\mathrm{NO}$ in inducing vascular bed adaptation. By contrast, 3 growth factors (VEGF, PDGF, and IGF-1) remained upregulated in our study, as previously reported. ${ }^{14-16}$ The involvement of ET in vascular function after pneumonectomy is less clear. Onizuka and colleagues ${ }^{17,18}$ described a transient increase in ET-1 levels after thoracic surgery in humans, with normalization within 72 hours. Although ET-1 expression was normal 5 weeks after pneumonectomy in our study, ETA and ETB expressions were decreased. This persistent downregulation of the ET pathway may have prevented pulmonary vascular remodeling after pneumonectomy. Indeed, in a previous study in pigs, we showed that pulmonary high flow induced by 5 weeks of aortopulmonary shunting resulted in distal pulmonary vasculopathy associated with ETA upregulation. Moreover, anti-ETA therapy accelerated the reversal of the flow-induced PA disease after flow correction. ${ }^{19}$ These data suggested a role for both the NO and the ET pathways in preventing vascular remodeling after pneumonectomy. They also indicated that pulmonary blood flow redistribution to nonobstructed PAs, inducing increases in shear stress and PA pressures, could not fully explain the development of distal pulmonary vasculopathy. By contrast, in CTEPH, pulmonary vascular resistances are not correlated to the degree of proximal anatomic obstruction as assessed by pulmonary angiography or perfusion nuclear scan. Indeed, a low anatomic obstruction (less than half of the PA bed) can be associated in some patients with high pulmonary resistance. ${ }^{9}$ This subgroup of patients with "out of proportion PH" are at high risk for surgery. Thus, there was substantial evidence supporting a major role for the ischemic lung in the development of pulmonary vascular remodeling in nonobstructed PAs with subsequent $\mathrm{PH}$.

In our model of right PA ligation, blood flow redistribution to the left lung combined with contralateral lung ischemia for 5 weeks resulted in pulmonary vasculopathy with increases in pulmonary vascular resistance and distal PA
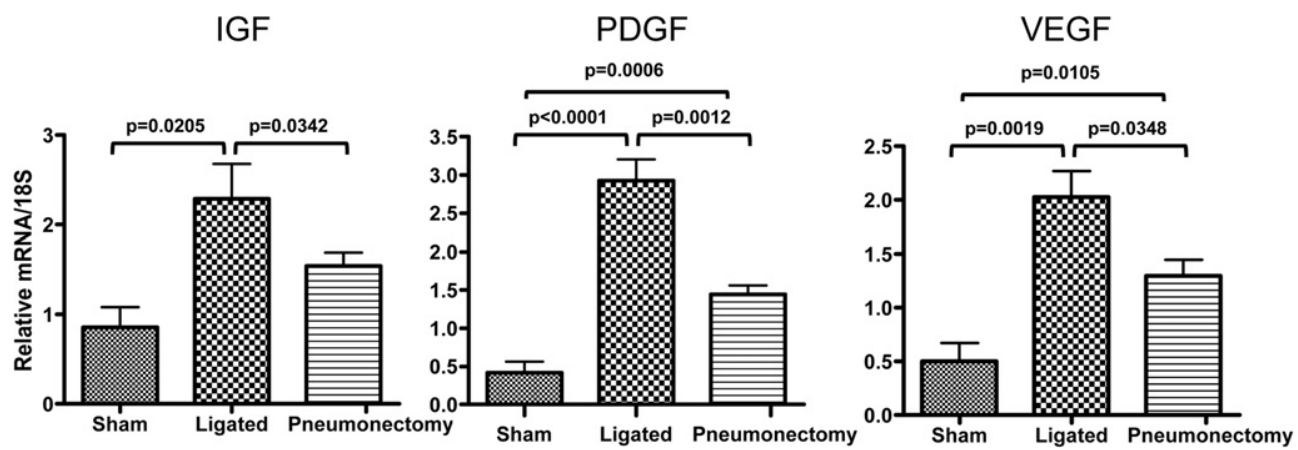

FIGURE 6. Quantification of mRNAs for IGF, PDGF, and VEGF in left lung parenchyma from the sham operation, ligation, and pneumonectomy groups. PDGF and VEGF gene expressions were increased in the pneumonectomy group compared with the sham group. Expression of these proangiogenic factors was greater in the ligated group than in the pneumonectomy and sham groups. Left lung IGF gene expression was increased in the ligated group compared with both the sham and the pneumonectomy groups. $I G F$, Insulin-like growth factor; $P D G F$, platelet-derived growth factor; $V E G F$, vascular endothelial growth factor. 
wall thickness, as well as impairment of endotheliumdependent vasorelaxation. Left lung ligation has been extensively used to study the mechanisms of postobstructive pulmonary vasculopathy. ${ }^{20} \mathrm{We}$ and others consistently found that chronic lung ischemia was associated with upregulation of angiogenic growth factors, leading to the development of an exuberant systemic blood supply to the ischemic lung. ${ }^{20-22}$ However, our present finding of contralateral vasculopathy had not been documented previously in the PA ligation model. This finding is not surprising given the marked increases in the expressions of 3 important proangiogenic factors, namely, VEGF, PDGF, and IGF, in the nonischemic lung 5 weeks after right PA ligation. These increases suggest that the ischemic lung may release endocrine factors that stimulate the expression of these 3 genes. Our results are consistent with previously reported studies on the role of proangiogenic factors in the development of pulmonary vasculopathy. Thus, VEGF was strongly expressed in pulmonary angioproliferative plexiform lesions from patients with severe idiopathic or secondary $\mathrm{PH} .{ }^{23}$ Likewise, our group showed previously that PDGF played a major role in the proliferation and migration of PA SMCs in human $\mathrm{PH} .{ }^{24}$ IGF is also known as an important activator of PA SMC proliferation. ${ }^{25}$ These peptides may constitute targets for treatments designed to prevent pulmonary vasculopathy in selected patients with severe CTEPH.

\section{CONCLUSIONS}

Chronic lung ischemia plays a key role in the development of pulmonary vasculopathy in nonobstructed pulmonary territories. In our study, pulmonary vasculopathy was absent in the remaining lung 5 weeks after pneumonectomy but developed in the nonobstructed pulmonary territories 5 weeks after right PA ligation, suggesting that factors released by the ischemic lung induced vascular remodeling in the contralateral lung. These endocrine factors may be factors involved in vascular SMC proliferation.

\section{References}

1. Mercier O, Sage E, de Perrot M, Tu L, Marcos E, Decante B, et al. Regression of flow-induced pulmonary arterial vasculopathy after flow correction in piglets. $J$ Thorac Cardiovasc Surg. 2009;137:1538-46.

2. Muller WJ, Dammann JJ, Head WJ. Changes in the pulmonary vessels produced by experimental pulmonary hypertension. Surgery. 1953;34:363-75.

3. Rendas A, Lennox S, Reid L. Aorta-pulmonary shunts in growing pigs. Functional and structural assessment of the changes in the pulmonary circulation. $J$ Thorac Cardiovasc Surg. 1979;77:109-18.
4. Fike C, Kaplowitz M, Bousamra MN. eNOS and prostanoid enzymes in lungs of newborn piglets with chronic aortopulmonary shunts. Am J Physiol Lung Cell Mol Physiol. 2001;281:L475-82.

5. Fadel E, Mazmanian G, Baudet B, Detruit H, Verhoye J, Cron J, et al. Endothelial nitric oxide synthase function in pig lung after chronic pulmonary artery obstruction. Am J Respir Crit Care Med. 2000;162(4 Pt 1):1429-34.

6. Rondelet B, Kerbaul F, Motte S, van Beneden R, Remmelink M, Brimioulle S, et al. Bosentan for the prevention of overcirculation-induced experimental pulmonary arterial hypertension. Circulation. 2003;107:1329-35.

7. Moser K, Auger W, Fedullo P. Chronic major-vessel thromboembolic pulmonary hypertension. Circulation. 1990;81:1735-43.

8. Peacock A, Simonneau G, Rubin L. Controversies, uncertainties and future research on the treatment of chronic thromboembolic pulmonary hypertension. Proc Am Thorac Soc. 2006;3:608-14.

9. Dartevelle P, Fadel E, Mussot S, Chapelier A, Herve P, de Perrot M, et al. Chronic thromboembolic pulmonary hypertension. Eur Respir J. 2004;23:637-48.

10. Hoeper MM, Rubin LJ. Update in pulmonary hypertension 2005. Am J Respir Crit Care Med. 2006;173:499-505.

11. Maxey T, Fernandez L, Reece T, Keeling W, Kron I, Laubach V. Endothelial nitric oxide synthase is essential for postpneumonectomy compensatory vasodilation. Ann Thorac Surg. 2006;81:1234-8.

12. Johnson RJ, Cassidy S, Grover R, Ramanathan M, Estrera A, Reynolds R, et al Effect of pneumonectomy on the remaining lung in dogs. J Appl Physiol. 1991; 70:849-58.

13. Friedli B, Kent G, Kidd B. The effect of increased pulmonary blood flow on the pulmonary vascular bed in pigs. Pediatr Res. 1975;9:547-53.

14. McAnulty RJ, Guerreiro D, Cambrey AD, Laurent GJ. Growth factor activity in the lung during compensatory growth after pneumonectomy: evidence of a role for IGF-1. Eur Respir J. 1992;5:739-47.

15. Yuan S, Hannam V, Belcastro R, Cartel N, Cabacungan J, Wang J, et al. A role for platelet-derived growth factor-BB in rat postpneumonectomy compensatory lung growth. Pediatr Res. 2002;52:25-33.

16. Jancelewicz T, Grethel EJ, Chapin CJ, Clifton MS, Nobuhara KK. Vascular endothelial growth factor isoform and receptor expression during compensatory lung growth. J Surg Res. 2010;160:107-13.

17. Onizuka M, Miyauchi T, Mitsui K, Suzuki N, Masaki T, Goto K, et al. Endothelin-1 mediates regional blood flow during and after pulmonary operations. J Thorac Cardiovasc Surg. 1992;104:1696-701.

18. Onizuka M, Miyauchi T, Morita R, Akaogi E, Mitsui K, Suzuki N, et al. Increased plasma concentrations of endothelin-1 during and after pulmonary surgery. J Cardiovasc Pharmacol. 1991;17(Suppl 7):S402-3.

19. Mercier O, Sage E, Izziki M, Humbert M, Dartevelle P, Eddahibi S, et al. Endothelin A receptor blockade improves regression of flow-induced pulmonary vasculopathy in piglets. $J$ Thorac Cardiovasc Surg. 2010;140:677-83.

20. Fadel E, Michel R, Eddahibi S, Bernatchez R, Mazmanian G, Baudet B, et al. Regression of postobstructive vasculopathy after revascularization of chronically obstructed pulmonary artery. J Thorac Cardiovasc Surg. 2004;127:1009-17.

21. Charan NB, Carvalho P. Angiogenesis in bronchial circulatory system after unilateral pulmonary artery obstruction. J Appl Physiol. 1997;82:284-91.

22. Michel RP, Hakim TS. Increased resistance in postobstructive pulmonary vasculopathy: structure-function relationships. J Appl Physiol. 1991;71:601-10.

23. Voelkel N, Vandivier R, Tuder R. Vascular endothelial growth factor in the lung. Am J Physiol Lung Cell Mol Physiol. 2006;290:L209-21.

24. Perros F, Montani D, Dorfmuller P, Durand-Gasselin I, Tcherakian C, Le Pavec J, et al. Platelet-derived growth factor expression and function in idiopathic pulmonary arterial hypertension. Am J Respir Crit Care Med. 2008; 178:81-8.

25. Dempsey EC, Stenmark KR, McMurtry IF, O'Brien RF, Voelkel NF Badesch DB. Insulin-like growth factor I and protein kinase $\mathrm{C}$ activation stimulate pulmonary artery smooth muscle cell proliferation through separate but synergistic pathways. J Cell Physiol. 1990;144:159-65. 